\title{
Vortex matter in layered superconductors without Josephson coupling: numerical simulations within a mean-field approach
}

\author{
Hans Fangohr \\ School of Engineering Sciences and Department of Physics and Astronomy, \\ University of Southampton, Southampton, SO17 1BJ, United Kingdom \\ Alexei E. Koshelev \\ Materials Science Division, Argonne National Laboratory \\ 9700 South Cass Avenue, Argonne, Illinois 60439, United States. \\ Matthew J. W. Dodgson \\ Theory of Condensed Matter Group, Cavendish Laboratory \\ University of Cambridge, Madingley Road, Cambridge, CB3 OHE, United Kingdom
}

\begin{abstract}
We study vortex matter in layered superconductors in the limit of zero Josephson coupling. The long range of the interaction between pancake vortices in the $c$-direction allows us to employ a mean-field method: all attractive inter-layer interactions are reduced to an effective substrate potential, which pancakes experience in addition to the same-layer pancake repulsion. We perform numerical simulations of this mean-field model using two independent numerical implementations with different simulation methods (Monte-Carlo sampling and Langevin molecular dynamics). The substrate potential is updated self-consistently from the averaged pancake density. Depending on temperature, this potential converges to a periodic profile (crystal) or vanishes (liquid). We compute thermodynamic properties of the system, such as the melting line, the instability line of the crystal, and the entropy jump across the melting transition. The simulation results are in good agreement with approximate analytical calculations.

PACS numbers: 74.60.Ge, 74.80.Dm
\end{abstract}

\section{INTRODUCTION}

The vortex state in type-II superconductors is a complex physical system. Within the layered high-temperature materials, such as $\mathrm{Bi}_{2} \mathrm{Sr}_{2} \mathrm{CaCu}_{2} \mathrm{O}_{8}$ (BSCCO) or $\mathrm{YBa}_{2} \mathrm{Cu}_{3} \mathrm{O}_{7}$ (YBCO), vortex lines fan understood as wiggling stacks of pancake vortices. The thermodynamic properties of the vortex state are determined by the interaction between pancake vortices. There are two mechanisms of pancake interaction: (i) electromagnetic interaction and (ii) Josephson coupling. The electromagnetic interaction is mediated by supercurrents circulating around each pancake, whereas the Josephson coupling results from the energy cost due to a phase shift between the superconducting order parameters in the neighboring layers.

To understand the phase diagram of high-temperature superconductors and in particular the melting line of the vortex lattice, havior of vortex matter under a variety of experimental conditions. In moderately anisotropic materials, such as YBCO, the short-range Josephson coupling is the dominant inter-layer interaction and the vortices are well de-

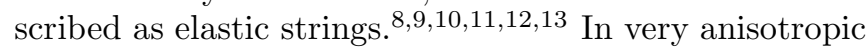
materials on the other hand, such as BSCCO, the Josephson coupling is weak, and the long-range electromagnetic interaction between the pancakes should be taken into account. In this paper we consider very anisotropic materials in the absence of Josephson coupling, and neglect pinning. Even after keeping only the electromagnetic coupling, the problem remains a challenging one, due to the long range of the interactions: the energy of electromagnetic interaction between two pancakes depends logarithmically on the separation along the layers and decays exponentially with the number of layers between the pancakes. More specifically, the interaction is repulsive between pancakes in the same layer, and attractive between pancakes in different layers, and the decay length of the exponential dependence is the London penetration depth $\lambda$, which is typically 100 times larger than the layer spacing $s$. Approximately, this system has been investigated within the density-functional theory $14,15,16$

For a numerical investigation of the system, one can in principle simulate directly a stack of two-dimensional (2D) pancake systems taking into account all of the interlayer interactions. However, the computational challenge is that the interlayer attraction between pancakes extends over a range of $2 \lambda / s \sim 100-150$ layers. In addition, realistic simulation of the melting transition requires at least several hundred point vortices per layer. So far, direct numerical investigations have been performed only on small systems using abut 10 layers and of the order of 100 vortices 1 1] $18,29.20122$ This is not sufficient to describe realistically the vortex state in BSCCO. With today's computational resources, it is not feasible to perform realistic direct three-dimensional (3D) simulations of this system because the necessary computational effort grows quadratically with the number of layers.

Fortunately, one can benefit from the long range of the interlayer coupling. As the interlayer force on a pancake 
(A)

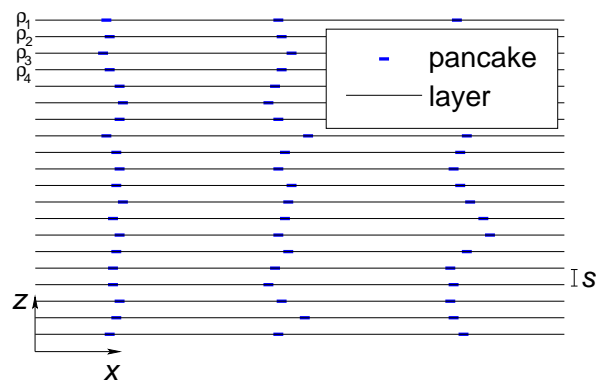

(B)

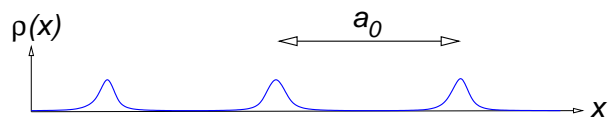

(C)

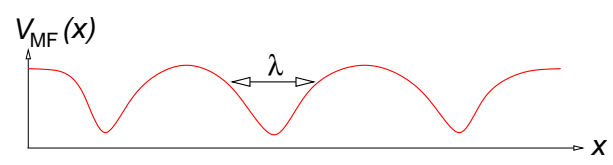

FIG. 1: Schematic representation of the substrate model. (A) The pancake positions $\rho_{n}(x)$ in each layer $n$, are $(B)$ averaged over the layers in order to obtain the averaged pancake density $\rho(x)$. From the average pancake density we compute $(C)$ the substrate potential $V_{\mathrm{MF}}(x)$, which is smeared over a length of the order of $\lambda$. The vortex lattice spacing is $a_{0}$ and $s$ is the layer spacing.

is the result of a sum of a large number $(\sim 2 \lambda / s)$ of small contributions, it can be calculated by a mean-field approach. The exact value of this force is determined by the instantaneous pancake densities in the large number of layers. In the crystal state the instantaneous density can be decomposed into the average density, which is a periodic function of the in-plane coordinates, and a fluctuating contribution. In the mean-field approach to the interlayer interactions, one replaces the instantaneous densities in the other layers by the average density. This approach gives a quantitatively correct description of the system, because due to the law of large numbers, the neglected force from the fluctuating densities is typically smaller than the average interlayer force by the factor $\sim \sqrt{s / \lambda} \ll 1$. The calculation then takes the form of independent layers, with the pancakes in each layer feeling an effective "substrate potential" 23 This substrate potential is the cumulative affect of the attraction of pancakes in all other layers as illustrated in Fig. 1. Pancakes within one layer interact directly with each other, whereas the interaction with pancakes in other layers is mediated via the substrate potential. Thus, each layer is treated individually, until a new substrate potential can be computed. This process is iterated, until the substrate has converged to a steady solution. In this paper, we present the first numerical implementations of this substrate model and show results which we compare with the semi-analytic approximations given in Ref. [23.

We summarize this work in Fig. 2. On the left the central idea is visualized: pancakes experience attractive inter-layer interactions through the substrate potential
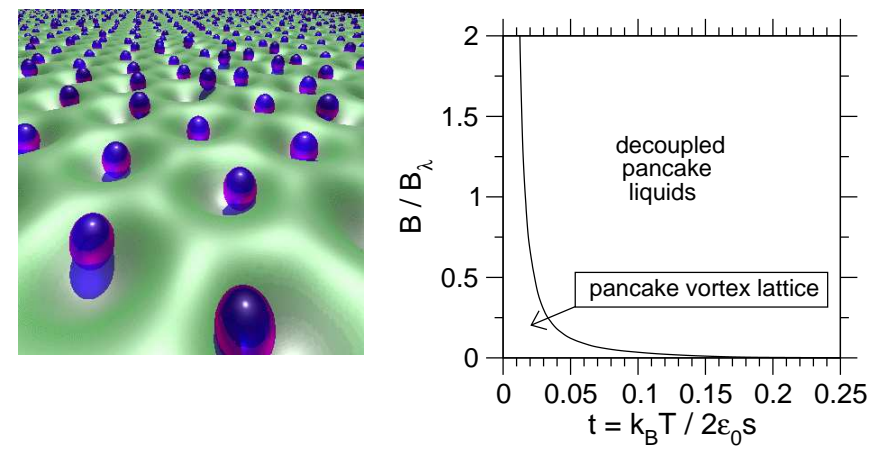

FIG. 2: Left: Snapshot of the pancakes (visualized by spheres) placed onto the substrate potential (visualized as a surface) at $B=B_{\lambda}$ and $t=1 / 59 \approx 0.017$ just below the melting transition to demonstrate how the substrate potential constrains the pancake motion. Right: The phase diagram we have computed using the substrate method.

which stabilizes the pancake crystal. On the right we show the computed melting line separating a 3D pancake vortex lattice from decoupled $2 \mathrm{D}$ liquids. We express magnetic induction in units of $B_{\lambda} \equiv \Phi_{0} / \lambda^{2}$, where $\Phi_{0}$ is the magnetic flux quantum, such that the pancakespacing in a triangular lattice is $a_{0}=\sqrt{2 / \sqrt{3}} \lambda \approx 1.07 \lambda$ at $B=B_{\lambda}$. We use a dimensionless temperature, $t$, which is the ratio of the thermal energy $k_{\mathrm{B}} T$ to the prefactor $2 s \epsilon_{0}$ of the logarithmic pancake-pancake interaction,

$$
t \equiv \frac{1}{\Gamma} \equiv \frac{k_{\mathrm{B}} T}{2 \epsilon_{0} s}
$$

where $\epsilon_{0}=\Phi_{0}^{2} /\left(4 \pi \mu_{0} \lambda^{2}\right), \mu_{0}$ is the vacuum permeability, and $s$ is the layer spacing. This allows to compare our results with outcomes from 2D one-component Coulomb plasma simulations, 242526 where frequently $\Gamma=1 / t$ is used to express temperatures. At low fields, the electromagnetic attraction of range $\lambda \gg s$ between pancakes in different layers stabilizes the 3D pancake-vortex lattice. Increasing the magnetic field decreases the relative strength of the inter-layer coupling. At high fields, $B \gg B_{\lambda}$, the long-range repulsive interaction within the layer dominates, and the $3 \mathrm{D}$ pancake lattice melts at a temperature close to the $2 \mathrm{D}$ melting temperature.

In Sec. II we describe the substrate model in detail, including three different methods for the efficient computation of the substrate potential (Sec. II C). The results, including the equilibrium phase diagram, are shown in Sec. III before we conclude in Sec. IV. The appendix gives a derivation of the correlation correction to the free energy, and shows that our mean-field approach should be accurate to order $s / \lambda$. 


\section{MEAN-FIELD APPROACH (SUBSTRATE MODEL)}

\section{A. The mean-field inter-layer coupling}

The in-layer energy $\mathcal{E}^{\text {in }}$ and the inter-layer energy $\mathcal{E}^{\text {inter }}$ of a system of electromagnetically interacting pancakes in a layered superconductor is, respectively,

$$
\mathcal{E}^{\text {in }}=\sum_{n} E_{n}^{\text {in }}=\sum_{n} \frac{1}{2} \sum_{j^{\prime} \neq j} U\left(\mathbf{R}_{j}^{n}-\mathbf{R}_{j^{\prime}}^{n}, 0\right)
$$

and

$$
\mathcal{E}^{\text {inter }}=\frac{1}{2} \sum_{n^{\prime} \neq n} \sum_{j, j^{\prime}} U\left(\mathbf{R}_{j}^{n}-\mathbf{R}_{j^{\prime}}^{n^{\prime}}, n-n^{\prime}\right) .
$$

Indices $n$ and $n^{\prime}$ count over layers, and $j$ and $j^{\prime}$ over pancakes in the layers, $\mathbf{R}_{j}^{n}$ is the $(2 \mathrm{D})$ position of pancake $j$ in layer $n$, and $U(\mathbf{R}, n)$ is the coupling energy for two pancakes separated by a vector $(\mathbf{R}, z)$, where $z=n s$, with $s$ being the layer spacing. The $z$-axis is chosen perpendicular to the layers.

The in-layer pancake interaction 1 is

$$
\begin{aligned}
U(\mathbf{r}, 0)=2 \epsilon_{0} s \quad & \left(\left(1-\frac{s}{2 \lambda}\right) \ln \left(\frac{L}{r}\right)\right. \\
& \left.+\frac{s}{2 \lambda} \int_{r}^{\infty} \mathrm{d} r^{\prime} \frac{\exp \left(-r^{\prime} / \lambda\right)}{r^{\prime}}\right)
\end{aligned}
$$

and the inter-layer interaction $(n \neq 0)$ is

$$
\begin{aligned}
U(\mathbf{r}, n)=- & \frac{\epsilon_{0} s^{2}}{\lambda}\left(\exp \left(-\frac{n s}{\lambda}\right) \ln \left(\frac{L}{r}\right)\right. \\
& \left.-\int_{r}^{\infty} \mathrm{d} r^{\prime} \frac{\exp \left(-\sqrt{r^{\prime 2}+(n s)^{2}} / \lambda\right)}{r^{\prime}}\right) .
\end{aligned}
$$

Using

$$
\rho_{n}(\mathbf{r})=\sum_{j} \delta\left(\mathbf{r}-\mathbf{R}_{j}^{n}\right)
$$

we rewrite

$$
\mathcal{E}^{\text {inter }}=\frac{1}{2} \sum_{n \neq n^{\prime}} \int \mathrm{d}^{2} r \mathrm{~d}^{2} r^{\prime} \rho_{n}(\mathbf{r}) \rho_{n^{\prime}}\left(\mathbf{r}^{\prime}\right) U\left(\mathbf{r}-\mathbf{r}^{\prime}, n-n^{\prime}\right)
$$

We separate pancake density fluctuations from the layeraverage density

$$
\begin{aligned}
\rho(\mathbf{r}) & \equiv\left\langle\rho_{n}(\mathbf{r})\right\rangle, \\
\rho_{n}(\mathbf{r}) & =\rho(\mathbf{r})+\delta \rho_{n}(\mathbf{r}),
\end{aligned}
$$

and obtain from (7)

$$
\begin{aligned}
\mathcal{E}^{\text {inter }}= & \frac{1}{2} \sum_{n \neq n^{\prime}} \int \mathrm{d}^{2} r \mathrm{~d}^{2} r^{\prime} U\left(\mathbf{r}-\mathbf{r}^{\prime}, n-n^{\prime}\right) \\
& \times\left[\rho(\mathbf{r}) \rho\left(\mathbf{r}^{\prime}\right)+2 \rho\left(\mathbf{r}^{\prime}\right) \delta \rho_{n}(\mathbf{r})+\delta \rho_{n}(\mathbf{r}) \delta \rho_{n^{\prime}}\left(\mathbf{r}^{\prime}\right)\right] .
\end{aligned}
$$

Because the difference $n-n^{\prime}$ in the last sum extends over a very large number of layers $(\sim \lambda / s)$, a typical value of the sum $\sum_{n^{\prime}} U\left(\mathbf{r}-\mathbf{r}^{\prime}, n-n^{\prime}\right) 2 \rho\left(\mathbf{r}^{\prime}\right)$ is larger than a typical value of the sum $\sum_{n^{\prime}} U\left(\mathbf{r}-\mathbf{r}^{\prime}, n-n^{\prime}\right) \delta \rho_{n^{\prime}}$ by the factor $\sim \sqrt{\lambda / s}$. Again, the law of large numbers allows us to neglect the last term in (10) leading to the mean-field description of the interlayer interactions. A more precise justification is given in the Appendix, where the free energy correction due to the correlation term is shown to be smaller than the mean-field free energy by the factor $s / \lambda$.

Separating the pancake density into the average value $\rho=\left\langle\rho_{n}(\mathbf{r})\right\rangle$ and a modulating part, we can split the total magnetic coupling energy into two parts, each with a quite different meaning. The part containing the average density does not depend on temperature and formally diverges due to the logarithmic term in (5). This divergence exactly compensates a similar divergence in the in-plane energy. Within the mean-field approach the part of the coupling energy sensitive to density variations is finite only in the crystal state. In the liquid state it vanishes.

For the mean-field interlayer energy $\mathcal{E}^{\mathrm{MF}}$ we obtain from 10

$$
\begin{aligned}
& \mathcal{E}^{\mathrm{MF}}=\sum_{n} E_{n}^{\mathrm{MF}} \\
& =\frac{1}{2} \sum_{n \neq n^{\prime}} \int \mathrm{d}^{2} r \mathrm{~d}^{2} r^{\prime} U\left(\mathbf{r}-\mathbf{r}^{\prime}, n-n^{\prime}\right) \\
& \quad \times\left[\rho(\mathbf{r}) \rho\left(\mathbf{r}^{\prime}\right)+2 \rho\left(\mathbf{r}^{\prime}\right) \delta \rho_{n}(\mathbf{r})\right] \\
& =\frac{1}{2} \sum_{n} \int \mathrm{d}^{2} r V_{\mathrm{MF}}(\mathbf{r}) \rho(\mathbf{r})+\sum_{n} \int \mathrm{d}^{2} r V_{\mathrm{MF}}(\mathbf{r}) \delta \rho_{n}(\mathbf{r}) \\
& \underline{\underline{9}} \quad-\frac{1}{2} \sum_{n} \int \mathrm{d}^{2} r V_{\mathrm{MF}}(\mathbf{r}) \rho(\mathbf{r})+\sum_{n} \int \mathrm{d}^{2} r V_{\mathrm{MF}}(\mathbf{r}) \rho_{n}(\mathbf{r}) .
\end{aligned}
$$

The last term describes fluctuations in the fixed substrate potential $V_{\mathrm{MF}}$,

$$
\begin{aligned}
V_{\mathrm{MF}}(\mathbf{r}) & =\int \mathrm{d}^{2} r^{\prime}\left[\sum_{n \neq 0} U\left(\mathbf{r}-\mathbf{r}^{\prime}, n\right)\right] \rho\left(\mathbf{r}^{\prime}\right) \\
& =\int \mathrm{d}^{2} r^{\prime} \mathcal{U}\left(\mathbf{r}-\mathbf{r}^{\prime}\right) \rho\left(\mathbf{r}^{\prime}\right) \\
& \equiv(\mathcal{U} * \rho)(\mathbf{r})
\end{aligned}
$$

with

$$
\mathcal{U}(\mathbf{r}) \equiv \sum_{n \neq 0} U(\mathbf{r}, n)
$$

$\mathcal{U}(\mathbf{r})$ is the interaction potential of a pancake separated by $\mathbf{r}$ from a stack of pancakes minus the interaction of the (missing) pancake in the same layer and is given by

$$
\mathcal{U}(\mathbf{r})=2 \epsilon_{0} s K_{0}\left(\frac{r}{\lambda}\right)-U(\mathbf{r}, 0)
$$


with $K_{0}(x)$ being a modified Bessel function of the second kind. Ignoring terms of the order of $s / 2 \lambda$, the pancakepancake repulsion (4) simplifies to

$$
U(\mathbf{r}, 0)=2 \epsilon_{0} s \ln \left(\frac{L}{r}\right) .
$$

In our calculations we find it useful to use the form in Fourier space,27

$$
\begin{aligned}
\mathcal{U}(\mathbf{q}) & =4 \pi \epsilon_{0} s\left(\frac{1}{\lambda^{-2}+q^{2}}-\frac{1}{q^{2}}\right) \\
& =-4 \pi \epsilon_{0} s \frac{\lambda^{-2}}{q^{2}\left(\lambda^{-2}+q^{2}\right)} .
\end{aligned}
$$

\section{B. Algorithm}

In principle, the substrate model can be implemented as follows:

1. Assume initial pancake densities $\rho_{n}(\mathbf{r})$, for example a hexagonal lattice in each layer $n$.

2. Average the pancake density $\rho_{n}(\mathbf{r})$ over all layers to obtain $\rho(\mathbf{r})$, (8).

3. Compute the substrate potential $V_{\mathrm{MF}}(\mathbf{r})$, (16), by convoluting the substrate interaction kernel $\mathcal{U}(\mathbf{r})$, (18), with the average pancake density $\rho(\mathbf{r})$

$$
V_{\mathrm{MF}}(\mathbf{r})=(\mathcal{U} * \rho)(\mathbf{r}) \text {. }
$$

4. For each layer $n$ compute the pancake distribution $\rho_{n}(\mathbf{r})$ using Monte Carlo or Langevin dynamics simulations. The total energy for layer $n$ contains the direct pancake-pancake interaction within the layer (2)

$$
E_{n}^{\mathrm{in}}=\frac{1}{2} \sum_{j^{\prime} \neq j} U\left(\mathbf{R}_{j}^{n}-\mathbf{R}_{j^{\prime}}^{n}, 0\right),
$$

and the relevant interaction with pancakes in other layers via the substrate potential (13)

$$
\begin{aligned}
& E_{n}^{\mathrm{MF}}-\underbrace{\frac{1}{2} \int \mathrm{d}^{2} r V_{\mathrm{MF}}(\mathbf{r}) \rho(\mathbf{r})}_{E^{\text {coup }}}+\int \mathrm{d}^{2} r V_{\mathrm{MF}}(\mathbf{r}) \rho_{n}(\mathbf{r}) \\
& \text { (6) }-E^{\text {coup }}+\sum_{j} V_{\mathrm{MF}}\left(\mathbf{R}_{j}^{n}\right) \text {, }
\end{aligned}
$$

$E^{\text {coup }}$ is constant for a given $\rho(\mathbf{r})$ and can therefore be ignored within the Monte Carlo/Langevin simulation as it only shifts the energy scale.

5. Go to 2, until $V_{\mathrm{MF}}$ (or $\rho$ ) has converged.
Since the substrate potential $V_{\mathrm{MF}}$ in step 4 is the same for all layers, we can compute $\rho_{n}(\mathbf{r})$ for many Langevindynamics time-steps (or Monte-Carlo sweeps) rather than many layers. Therefore, in order to obtain the averaged pancake density $\rho(\mathbf{r})$ in step 2, we average over time-steps (or sweeps) computed in one layer rather than averaging over layers.

Using the substrate potential, we reduce the solution of the $3 \mathrm{D}$ problem to performing one $2 \mathrm{D}$ simulation in the presence of the iteratively refined substrate potential.

\section{Numerical implementation}

We exploit the convolution theorem and compute the substrate potential in Fourier-space 27

$$
\begin{aligned}
V_{\mathrm{MF}}(\mathbf{r}) \stackrel{16)}{=}(\mathcal{U} * \rho)(\mathbf{r}) \\
=\int \frac{\mathrm{d}^{2} q}{(2 \pi)^{2}} \mathcal{U}(\mathbf{q}) \rho(\mathbf{q}) \exp (i \mathbf{q} \cdot \mathbf{r})
\end{aligned}
$$

using the analytical Fourier transform $\mathcal{U}(\mathbf{q})$ as given in (21), and the numerically computed

$$
\rho(\mathbf{q})=\int \mathrm{d}^{2} r \rho(\mathbf{r}) \exp (-i \mathbf{q} \cdot \mathbf{r}) .
$$

This has two advantages: firstly, we do not cut off the interaction kernel $\mathcal{U}$ within the simulation cell as would be the case in the real-space convolution. Secondly, this is numerically more efficient than performing the convolution (15) directly.

We have used three different methods for computing $V_{\mathrm{MF}}(\mathbf{r})$ numerically.

\section{The full method}

The "full method" computes the substrate potential $V_{\mathrm{MF}}$ using the full spectrum $\rho(\mathbf{q})$ of Fourier-components of the average pancake density $\rho(\mathbf{r})$ as shown in $(26)$. In our simulations we use a resolution of $\approx 100^{2}$ gridcells per pancake in order to compute $\rho(\mathbf{r})$ as an average over time-steps/sweeps. This results in reciprocal lattice vectors up to magnitudes of $\approx 100 Q_{0}$, where $Q_{0}=4 \pi /\left(\sqrt{3} a_{0}\right)$, because $\left|\mathbf{Q}_{\max }\right| / Q_{0} \approx 2 \pi /\left(\Delta x Q_{0}\right) \approx$ $a 0 / \Delta x \approx 100$. The necessary discrete Fourier-transform of $\rho(\mathbf{r})$, and the inverse transform of $V_{\mathrm{MF}}(\mathbf{q})=\mathcal{U}(\mathbf{q}) \rho(\mathbf{q})$ can be done efficiently ming an implementation of the Fast Fourier Transform.28

We pre-compute the substrate potential $V_{\mathrm{MF}}(\mathbf{r})$ on a mesh and interpolate subsequently for intermediate pancake positions while performing Langevin dynamics in the fixed substrate. We compute a new substrate every 200,000 time-steps. It is important to average over so many time-steps to reduce density fluctuations (due to poor statistics) in the pancake histogram, which would result in a deformed substrate potential. 
Note that $\rho(\mathbf{r})$ and $\rho(\mathbf{q})$ are discretized out of numerical necessity to compute a histogram but not for conceptual reasons.

\section{The Fourier-filtered method}

The average density $\rho(\mathbf{r})$ should be a periodic function, which can be represented by a discrete set of Fourier components. Therefore, the second method uses only a subset $\mathbf{Q}_{\mu}$ of the Fourier components $\mathbf{q}$ to represent $\rho(\mathbf{q})$

$$
\rho^{\mathrm{FF}}(\mathbf{q})=(2 \pi)^{2} \sum_{\mu} \rho_{\mathbf{Q}_{\mu}} \delta^{2}\left(\mathbf{q}-\mathbf{Q}_{\mu}\right)
$$

which we determine from the maxima of the structure factor and

$$
\rho_{\mathbf{Q}}=\left\langle\frac{1}{L_{x} L_{y}} \sum_{j} \exp \left(-i \mathbf{R}_{j}^{c} \cdot \mathbf{Q}\right)\right\rangle_{c}
$$

with $L_{x} L_{y}$ being the area of the simulation cell. We average over a set of configurations $c$ of pancake positions $\mathbf{R}_{j}^{c}$ (either sweeps or time-steps) to compute $\rho_{\mathbf{Q}}$.

Using $\rho^{\mathrm{FF}}(\mathbf{r})=(2 \pi)^{-2} \int \mathrm{d}^{2} q \rho^{\mathrm{FF}}(\mathbf{q}) \exp (i \mathbf{q} \cdot \mathbf{r})$ to present $\rho(\mathbf{r})$, we Fourier-filter $\rho(\mathbf{r})$, and keep only the relevant components for the computation of the periodic substrate. We can write

$$
\begin{aligned}
V_{\mathrm{MF}}^{\mathrm{FF}}(\mathbf{r}) & \stackrel{26}{=} \frac{\mathrm{d}^{2} q}{(2 \pi)^{2}} \mathcal{U}(\mathbf{q}) \rho^{\mathrm{FF}}(\mathbf{q}) \exp (i \mathbf{q} \cdot \mathbf{r}) \\
& \stackrel{\text { 26) }}{ } \sum_{\mu} \mathcal{U}\left(\mathbf{Q}_{\mu}\right) \rho_{\mathbf{Q}_{\mu}} \exp \left(i \mathbf{Q}_{\mu} \cdot \mathbf{r}\right) \\
& -4 \pi \epsilon_{0} s \sum_{\mu} \frac{\rho_{\mathbf{Q}_{\mu}} \exp \left(i \mathbf{Q}_{\mu} \cdot \mathbf{r}\right)}{Q_{\mu}^{2}\left(1+\lambda^{2} Q_{\mu}^{2}\right)}
\end{aligned}
$$

This is equivalent to using the full-method, but setting $\rho(\mathbf{Q})=0$ if $\mathbf{Q} \notin\left\{\mathbf{Q}_{\mu}\right\}$.

The advantage of the Fourier-filtered method is that we need to average over less iterations before we can compute a new pancake density, and subsequently a new substrate, because the substrate is per construction periodic. Using the Fourier-filtered method we use 500 timesteps/sweeps for each substrate iteration.

It turns out that it is not necessary to take the average (29) over different configurations but it is sufficient to use just one configuration (i.e. one time step or sweep):

$$
\rho_{\mathbf{Q}}=\frac{1}{L_{x} L_{y}} \sum_{j} \exp \left(-i \mathbf{R}_{j} \cdot \mathbf{Q}\right)
$$

Nevertheless, we run a simulation for 500 timesteps/sweeps with the same fixed substrate potential to reduce re-computation of $\rho_{\mathbf{Q}}$, and to give the pancakes some time to explore the system with a new substrate potential.

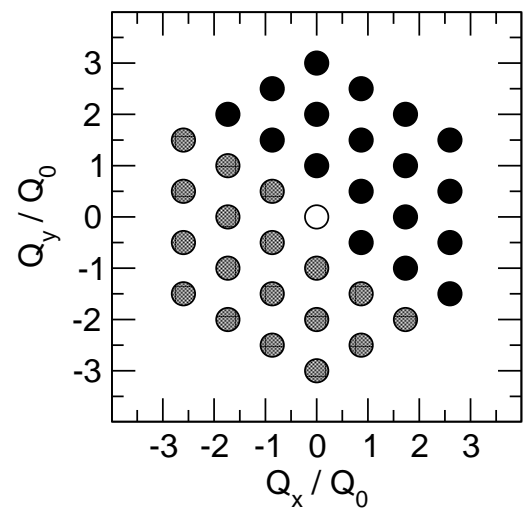

FIG. 3: Set of $\mathbf{Q}_{\mu}$-vectors up to third order (i.e. three "shells" around the origin) in reciprocal space used in the small-harmonics Fourier-filtered method to compute $\rho_{\mathbf{Q}_{\mu}}$. Due to the reality of $\rho(\mathbf{r})$ we have $\rho(\mathbf{q})=\bar{\rho}(-\mathbf{q})$ and it is therefore sufficient to compute only half of the 36 coefficients $\rho_{\mathbf{Q}_{\mu}}$.

\section{The small-harmonics (Fourier-filtered) approximation}

In addition to Fourier-filtering $\rho(\mathbf{r})$ we can speed up the computation further because close to the melting temperature, $\rho_{\mathbf{Q}_{\mu}}$ decays quickly for higher-order $\mathbf{Q}_{\mu}$ due to the Debye-Waller factor. We can estimate the reduction of $\rho_{\mathbf{Q}}$ due to the Debye-Waller factor

$$
\exp \left(-\frac{\left\langle u^{2}\right\rangle Q^{2}}{4}\right)=\exp \left(-\frac{1}{4} \frac{\left\langle u^{2}\right\rangle}{a_{0}^{2}} \frac{16 \pi^{2} Q^{2}}{Q_{0}^{2}}\right)
$$

where $Q_{0}=4 \pi /\left(\sqrt{3} a_{0}\right)$ and $\left\langle u^{2}\right\rangle$ is the mean-squared fluctuation displacement. Depending on $\left\langle u^{2}\right\rangle$ we can ignore all $\rho\left(\mathbf{Q}_{\mu}\right)$ with $\left|\mathbf{Q}_{\mu}\right|>Q^{\prime}$. For all but the smallest fields, we find close to the melting transition $\left\langle u^{2}\right\rangle / a_{0}^{2} \approx 0.02-0.03$ (see Section IIIE), and it is sufficient to include up to $3^{\text {rd }}$-order vectors $\mathbf{Q}_{\mu}$ in the summation in (32) as shown in Fig. 3 .

For the small-harmonics Fourier-filtered method it is more efficient to evaluate (32) for each pancake position occurring in the Langevin/Monte-Carlo simulation rather than pre-computing $V_{\mathrm{MF}}$ on a mesh.

We demonstrate the equivalence of the full and the Fourier-filtered method for the determination of the instability line in Sec. III A, and we compare with the small-harmonics Fourier-filtered method in Sec. III C.

\section{Monte Carlo and Langevin dynamics simulations}

We have two independent implementations of the small-harmonics Fourier-filtered method: AEK has written a Monte Carlo simulation that is based on energy evaluations, and HF has implemented a Langevin dynamics simulation based on force calculations. The results of both implementations agree perfectly. 


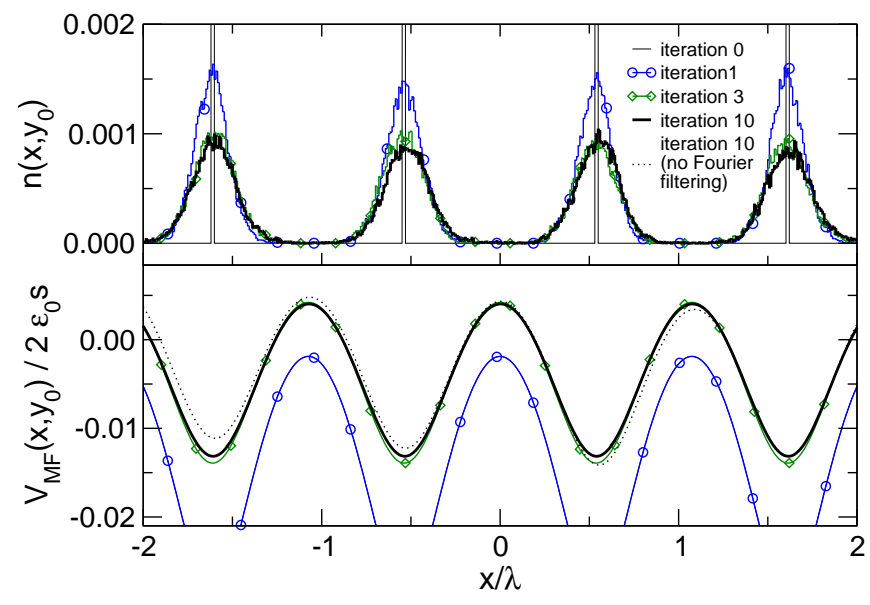

FIG. 4: Convergence to pancake lattice at $B=B_{\lambda}$ and $t=1 / 59 \approx 0.017$. Top: pancake histogram $n\left(x, y_{0}\right)$ taken along $y=y_{0}$. Bottom: substrate potential $V_{\mathrm{MF}}\left(x, y_{0}\right)$.

We follow standard vortex-state simulation techniques 290 including periodic boundary conditions for the in-plane interactions. We use a smooth cut-off for the vortex in-plane interactions.29.31 For the Langevin dynamics simulations, we compute the substrate forces numerically from the pre-computed mesh (Sec. II C 1) for the full method and the Fourierfiltered method. For the small-harmonics Fourier-filtered method we use the analytical derivative of (32). The Monte Carlo simulations were only implemented with the small-harmonics method. If not stated otherwise we use a system with 1020 pancakes.

\section{RESULTS}

\section{A. Time convergence of the substrate potential}

As described in Sec. IIB, we start each run with a hexagonal pancake distribution corresponding to zero temperature. Fig. 4 shows results for the Fourier-filtered method at $B=B_{\lambda}$ and at a temperature $t=1 / 59 \approx$ 0.017. The top plot shows a one-dimensional slice of the $2 \mathrm{D}$ pancake histogram $n\left(x, y_{0}\right)$ taken along $x$ at $y=y_{0}$. The histogram relates to the pancake density via $n(x, y)=\rho(x, y) \Delta x \Delta y$ where $\Delta x$ and $\Delta y$ are the spacings of the grid used to create the histogram. For the $0^{\text {th }}$ substrate-iteration we set the histogram to have narrow and high peaks at the pancake equilibrium positions corresponding to delta-peaks in a zero-temperature pancake density $\rho(\mathbf{r})$. Based on this initial pancake distribution, we compute the substrate potential, $V_{\mathrm{MF}}(\mathbf{r})$, for the first substrate-iteration, of which a one-dimensional slice at $y=y_{0}$ is shown in the lower part of Fig. 4. Using this substrate potential, we run the Langevin dynamics simulation for 200,000 steps which results in the histogram for iteration 1 as shown in the upper plot of Fig. 4.

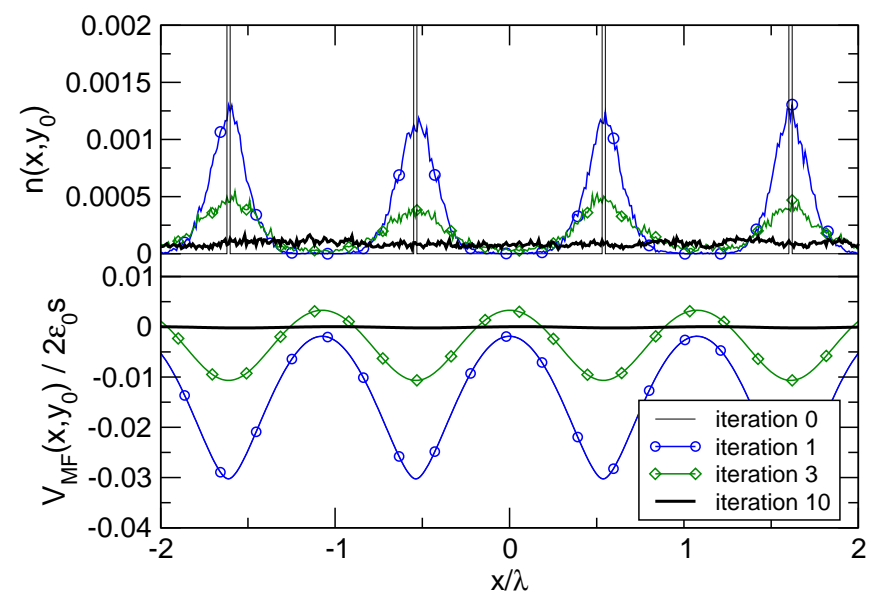

FIG. 5: Convergence to pancake liquid. As Fig. 1 but at $t=1 / 50=0.02$ above the melting temperature at $B=B_{\lambda}$.

Based on these data, we compute the substrate potential for iteration 2. We iterate the substrate re-computation until the substrate potential has reached a steady state (after typically than 10 substrate-iterations). The figure demonstrates that the system converges quickly to a pancake solid at this temperature below melting.

The dotted line in the lower part of Fig. 4 shows a comparison substrate potential for iteration 10 computed using the full method. While the amplitude and width of the wells (and thus the resulting force) are virtually identical to the Fourier-filtered data, the magnitude of the substrate from the full method varies slightly. This is due to (long wavelength) density fluctuations in the histogram data and reduces further if one uses more timesteps for each substrate iteration.

Fig. 5 shows data for $B=B_{\lambda}$ and a higher temperature $t=1 / 50=0.02$ which is above the melting temperature. Here, the pancake distribution broadens and consequently the substrate potential flattens quickly within the first few substrate iterations. Eventually, the system has become a disordered liquid with an approximately constant pancake density and the substrate is virtually flat, as shown for iteration 10 . We conclude that for this temperature and magnetic field the pancake lattice is unstable to melting into a pancake liquid.

For Fig. 4 and 5 we have used 200,000 time-steps for each substrate-iteration in order to be able to compare the full and the Fourier-filtered method, but it would be sufficient to use much less time-steps per substrateiteration for the Fourier-filtered methods. For production purposes, we use the small-harmonics Fourierfiltered method and update the substrate every 500 timesteps (Sec. II C 2). Although more substrate-iterations than with the full method are required before the system reaches a steady state, the small-harmonics Fourierfiltered approach is more efficient. The full method and both Fourier-filtered methods find that at $B=B_{\lambda}$ the pancake lattice becomes unstable for $0.017 \leq t \leq 0.018$. 


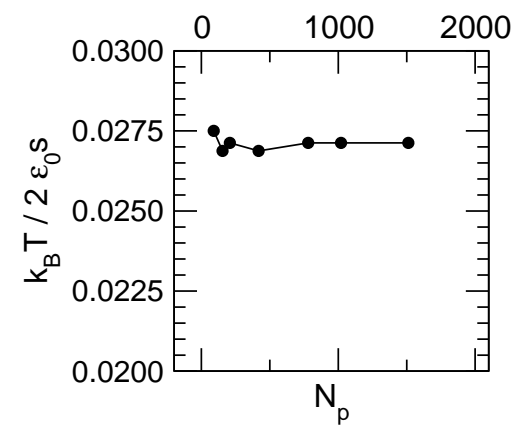

FIG. 6: Finite-size investigation of instability temperature at $B=0.4 B_{\lambda}$.

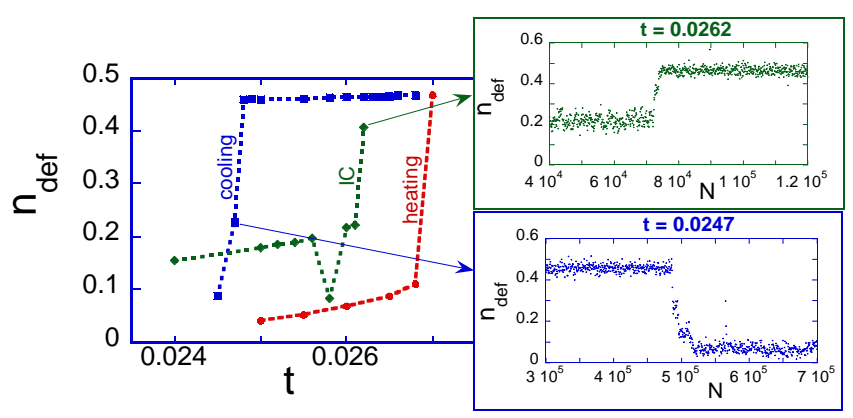

FIG. 7: Example of a hysteresis loop obtained by heating a crystal (circles) and cooling a liquid (squares) for $B=0.4 B_{\lambda}$. Each point was equilibrated for $9 \cdot 10^{5}$ Monte-Carlo steps. The crystal melts at $t=0.027$, while the liquid freezes at $t=0.0247$. Rhombs represent results obtained by simulations starting from the same intermediate defective configuration with $n_{\text {def }} \approx 0.2$, this configuration melts at $t=0.0262$, which we take as an estimate for the thermodynamic melting temperature. The insets show dependencies of the defect concentration on the Monte Carlo step at the temperatures where the intermediate configuration melts and the liquid configuration freezes. See also [32].

Fig. 6 shows how the instability temperature varies as a function of system size. For small numbers of vortices, $N_{\mathrm{p}}$, the temperature oscillates slightly and for larger systems it becomes constant. Most importantly, there is no general trend visible although the data ranges from $N_{\mathrm{p}}=90$ to $N_{\mathrm{p}}=1512$. This insensitivity to the system size demonstrates the local nature of the melting transition at this field.

\section{B. Hysteresis loop}

Rather than starting from a hexagonal crystal for every temperature, a better approach to determine the instability temperature is to subsequently increase $t$ until the system melts. We also find that by starting from a liquid configuration and lowering $t$, the system jumps into the crystal state at a certain freezing temperature. We

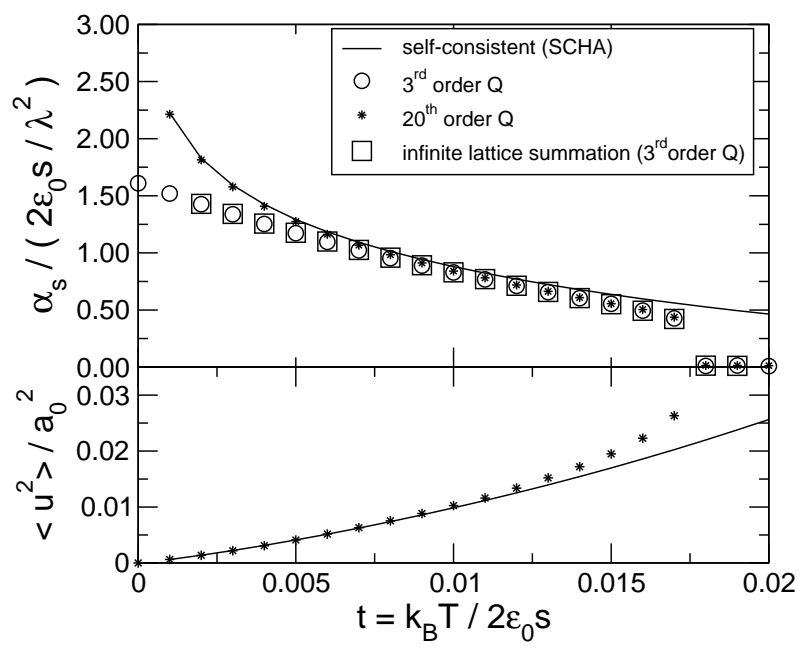

FIG. 8: Top: The substrate curvature $\alpha_{\mathrm{s}}$ at $B=B_{\lambda}$. Shown is our numerical solution of the Fourier-filtered method using $Q$-vectors up to $20^{\text {th }}$ order (stars), and using up to $3^{\text {rd }}$ order (circles). We have also shown results for $Q_{\max }=3 Q_{0}$ using an infinite lattice summation for the in-layer interaction (squares). Bottom: The pancake fluctuation width $\left\langle u^{2}\right\rangle$ (stars). For comparispm, we also show the results of a simple SCHA calculation 23 (solid line) of the softening of the substrate potential (not including thermal softening effects of the $2 \mathrm{D}$ lattice). The results are close to our numerical data at low temperatures, but as the melting point is approached there are extra (anharmonic) fluctuations in the simulation data for $\left\langle u^{2}\right\rangle$, resulting in an even softer substrate potential $\alpha_{\mathrm{s}}$.

expect the true melting temperature to lie within the instability and the freezing temperature. Such a hysteretic run is shown in Fig. 7.

In order to estimate the thermodynamic melting temperature at which the free energy of the solid and the liquid phase cross, we proceed as follows. Firstly, we store a vortex configuration taken from $2 \mathrm{D}$ melting-transition simulations. We chose a configuration from a timestep/sweep where the system was previously a solid but just starts melting, i.e. the defect density starts shooting up and the structure factor peaks start decaying. This vortex configuration is "intermediate" between a solid and a liquid. Secondly, we start the computation from this intermediate configuration (IC) for every temperature. The results for the IC simulations are shown in Fig. 7 (rhombs). We use the temperature at which the IC melts as a best approximation to the melting temperature of the physical 3D pancake-vortex lattice. 


\section{Temperature dependence of substrate curvature and pancake fluctuation width}

We can quantify the strength of the substrate potential with

$$
\alpha_{\mathrm{s}}=\frac{1}{N_{\mathrm{p}}} \sum_{j} \frac{\partial^{2}}{\partial x^{2}} V_{\mathrm{MF}}\left(\mathbf{R}_{j}-\mathbf{R}_{j}^{0}\right) .
$$

This is the curvature of the potential evaluated at deviations $\mathbf{R}_{j}-\mathbf{R}_{j}^{0}$ from the equilibrium lattice positions $\mathbf{R}_{j}^{0}$ and averaged over pancake positions $\mathbf{R}_{j}$. The second derivative can be taken analytically from (32).

Fig. 8 shows in the upper plot how $\alpha_{\mathrm{s}}$ varies with temperature. The solid line is an analytical prediction from treating the substrate softening due to thermal fluctuations within the self-consistent harmonic approximation (SCHA).23 All other data are simulation results from the Fourier-filtered method. The stars show $\alpha_{\mathrm{s}}$ computed using the small-harmonics Fourier-filtered method with $\mathbf{Q}_{\mu}$-vectors up to $20^{\text {th }}$ order. For low temperatures the data nearly coincide with the SCHA-solution. Close to melting the SCHA- $\alpha_{s}$ is larger than the numerical result. Therefore the simulations give a softer substrate and the lattice has larger thermal displacements. This difference could be due to the inadequacies of the SCHA which does not include the thermal softening of the 2D lattice. The more complex two-vertex self-consistent harmonic approximation (2VSCHA) does include these effects.23 The circles in Fig. 8 show results using $\mathbf{Q}_{\mu}$-vectors up to $3^{\text {rd }}$ order, as shown in Fig. 3. Close to the transition from solid to liquid around $t \approx 0.0175$ these data agree perfectly with the higher-order data. At lower temperatures the $3^{\text {rd }}$ order results deviate from the $20^{\text {th }}$ order because $\left\langle u^{2}\right\rangle$ becomes smaller in the Debye-Waller factor (34). However, as long as we are interested in temperatures close to the transition, the $3^{\text {rd }}$ order approach is sufficient.

The square boxes are computed using the $3^{\text {rd }}$ order approach, but instead of smoothly reducing the pancake interaction 31 at a distance of $\approx 7 a_{0}$, we use an infinite lattice summation technique for the logarithmic interaction. 33 This demonstrates that it is sufficient to use a (smooth) cut-off for the in-layer pancake interactions.

We compute the average pancake fluctuation width $\left\langle u^{2}\right\rangle$ by fitting to a distribution where each pancake is normally smeared around its equilibrium position $\mathbf{R}_{j}^{0}$

$$
\rho(\mathbf{r})=\frac{1}{2 \pi \sigma^{2}} \sum_{j} \exp \left(-\frac{\left|\mathbf{r}-\mathbf{R}_{\mathbf{j}}^{\mathbf{0}}\right|^{2}}{2 \sigma^{2}}\right) .
$$

The Fourier transform of $\rho(\mathbf{r})$ is

$$
\begin{aligned}
\rho(\mathbf{q}) & =\exp \left(\frac{-\sigma^{2} q^{2}}{2}\right) \sum_{j} \exp \left(-i \mathbf{q} \cdot \mathbf{R}_{j}^{0}\right) \\
& =(2 \pi)^{2} n_{0} \exp \left(\frac{-\sigma^{2} q^{2}}{2}\right) \sum_{\mu} \delta^{2}\left(\mathbf{q}-\mathbf{Q}_{\mu}\right) .
\end{aligned}
$$

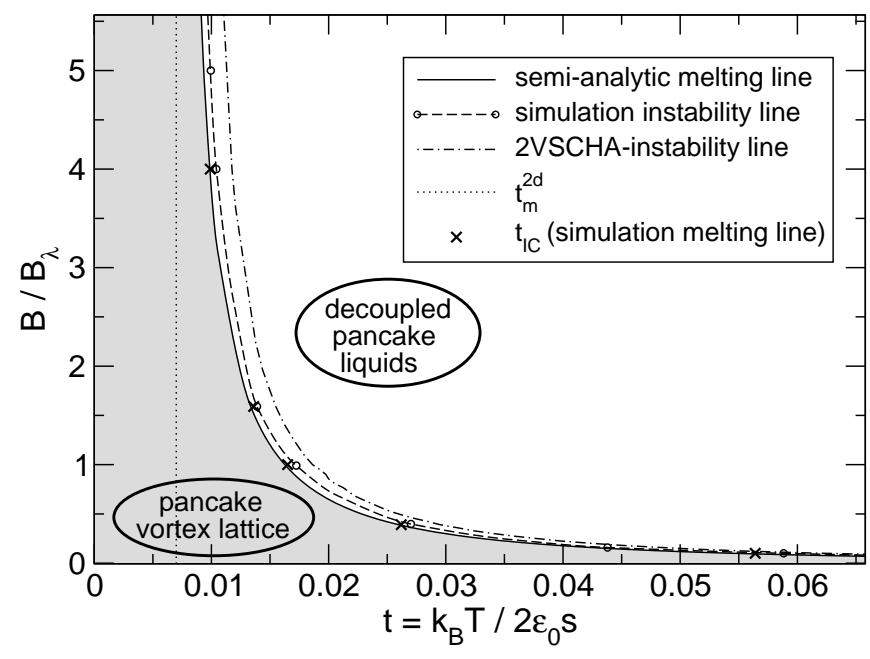

FIG. 9: Phase diagram of the electro-magnetically coupled 3D pancake system. Numerically computed instability line (black circles on dashed line) in comparison with the instability line from the 2VSCHA (dash-dotted line). Also shown is an semi-analytical estimate for the melting line from Ref. 23 , to be compared with our numerical estimate $t_{\mathrm{IC}}$ (crosses). We have shaded the solid phase underneath the melting in gray. The melting temperature $t_{m}^{2 \mathrm{~d}}=0.007$ of a $2 \mathrm{D}$ system is shown by a dotted line.

The Fourier components $\rho\left(\mathbf{Q}_{\mu}\right)$ have the Debye-Waller factor as an envelope, and by fitting a Gaussian to it, we can determine $\left\langle u^{2}\right\rangle=2 \sigma^{2}$.

The lower part of Fig. 8 shows computed values for $\left\langle u^{2}\right\rangle$. We express $\left\langle u^{2}\right\rangle$ in units of $a_{0}^{2}$ and it increases from 0 at zero temperature towards 0.028 close to the transition, which corresponds to a Lindemann number of $\approx 0.168$ at $B=B_{\lambda}$. In agreement with an overestimation of $\alpha_{\mathrm{s}}$ by the SCHA, $\left\langle u^{2}\right\rangle$ is underestimated in comparison with the numerical results close to the melting transition.

\section{Phase diagram}

As demonstrated in Sec. III A, we can determine for each parameter pair $(B, T)$ whether the pancake system remains a $3 \mathrm{D}$ pancake lattice, or whether it is unstable towards the liquid phase which consists of decoupled 2D liquids. (This is sometimes called a pancake gas, even though there are still very strong in-plane correlations in the decoupled layers. In the absence of Josephson coupling, a line-like liquid regime is expected only at extremely small magnetic fields.34)

We probe parameter space in the $B$ - $T$-plane as described in Sec. IIIA and compute an instability line for the phase diagram of the system, which is shown in Fig. 9 (circles on dashed line). We also show an estimate of the instability line that has been computed using the two-vertex self-consistent harmonic approximation (2VSCHA, dash-dotted) for the substrate model 23, 35 


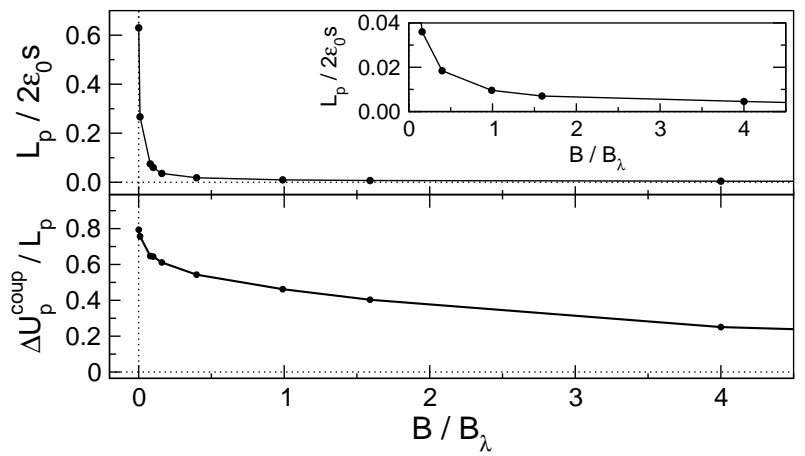

FIG. 10: Top: Latent heat $L_{\mathrm{p}}$ per pancake across the melting transition as a function of field. The inset shows the same data on a reduced scale. Bottom: Jump in inter-layer coupling energy, $\Delta U^{\text {coup }}=E^{\text {coup }}$, normalized by latent heat.

Since in this work we explicitly compute the pancake positions without using approximations (within the substrate model), we expect our result to be more accurate than the 2VSCHA. It can be seen that the 2VSCHA slightly overestimates the temperature for the instability line.

Our numerical estimates for the melting points at certain fields (see Section IIIB) are shown as the crosses in Fig. 9. Also shown as the solid line is the melting line calculated semi-analytically in Ref. 23. In this work the melting temperature was estimated by comparing approximate free energies $F=U-T S$ for the solid and liquid phases. The solid free energy was calculated from the SCHA, which gives a variational upper bound on the free energy. The liquid free energy was taken from earlier simulations of a single layer 25 i.e. it was assumed that the layers are completely uncoupled in the liquid state. Remarkably, our melting points from simulations lie on top of the semi-analytic line (to within our error bars).

For increasing fields $B$, the substrate becomes weaker and weaker and the melting temperature drops. In the limit of $B \rightarrow \infty$ we recover a $2 \mathrm{D}$ system with logarithmis interactions for which melting has been estimated $24,25,26$ to occur at $\Gamma_{\mathrm{m}}^{2 \mathrm{~d}} \approx 140 \pm 10 \Leftrightarrow t_{\mathrm{m}}^{2 \mathrm{~d}} \approx 0.007$, which is consistent with our results.

At low fields the pancake stacks are widely separated and interact only weakly with each other. In this limit the system melts below the pvaporation transition of an isolated stack of pancakes 36 at $\Gamma=4 \Leftrightarrow t=0.25$. In agreement with this, we find that the instability line approaches $t \approx 0.25$ for $B \rightarrow 0$ (see Fig. 2).

\section{E. Latent heat and jump in entropy}

We compute the latent heat per pancake, $L_{\mathrm{p}}$, by taking the difference of internal energy between the solid and the

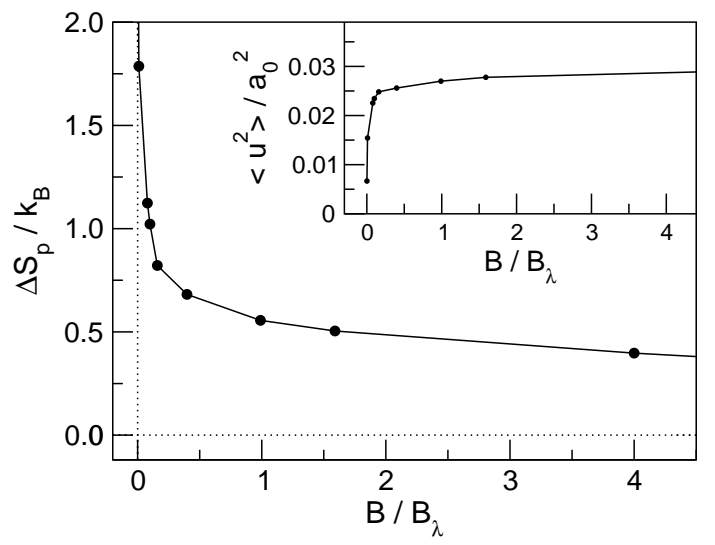

FIG. 11: Entropy jump $\Delta S_{p}$ per pancake as a function of field $B$. Inset: Pancake fluctuation width $\left\langle u^{2}\right\rangle$ normalized to $a_{0}^{2}$ at the melting point (the Lindemann number squared).

liquid phase at the melting temperature $T_{\mathrm{m}}$

$$
\begin{aligned}
L_{\mathrm{p}} & =\frac{1}{N_{\mathrm{p}}}\left(U_{\text {liquid }}-U_{\text {solid }}\right) \\
& =\frac{1}{N_{\mathrm{p}}}\left(E_{\text {liquid }}^{\text {in }}-\left(E_{\text {solid }}^{\text {in }}+E^{\text {coup }}\right)\right) .
\end{aligned}
$$

The internal energy $U$ of one layer in the solid phase consists of the in-plane energy $E^{\text {in }}$ (23) and the interlayer coupling energy $E^{\text {coup }}$, whereas $E^{\text {coup }}=0$ in the liquid phase in our model. In order to compute $E^{\text {coup }}$ for the solid phase, we use (12) where the second sum vanishes due to the definition of $\delta \rho_{n}$ :

$$
E^{\text {coup }}=\frac{1}{2} \int \mathrm{d}^{2} r V_{\mathrm{MF}}(\mathbf{r})[\rho(\mathbf{r})-\bar{\rho}],
$$

where $\bar{\rho}=\Phi_{0} / B$ is the mean density. For the Fourierfiltered methods

$$
\begin{gathered}
E^{\text {coup }} \stackrel{317}{\frac{1}{2} \int \mathrm{d}^{2} r} \sum_{\mu \neq 0} \mathcal{U}\left(\mathbf{Q}_{\mu}\right) \rho_{\mathbf{Q}_{\mu}} \exp \left(i \mathbf{Q}_{\mu} \cdot \mathbf{r}\right) \rho(\mathbf{r}) \\
\stackrel{27 .}{\frac{1}{2}} L_{x} L_{y} \sum_{\mu \neq 0} \mathcal{U}\left(\mathbf{Q}_{\mu}\right)\left|\rho_{\mathbf{Q}_{\mu}}\right|^{2} .
\end{gathered}
$$

For the full method, we have $\rho(\mathbf{r})$ as a histogram available, and we can integrate (39) numerically.

The top plot of Fig. 10 shows how the latent heat varies as a function of field. We have shown the jump in interlayer coupling energy normalized by the latent heat in the bottom part to demonstrate the contribution of the interlayer coupling to the latent heat. This plot shows that the substrate contribution to the latent heat dominates at low fields, and becomes less and less important towards high fields.

Fig. 11 shows the entropy jump across the transition, $\Delta S_{\mathrm{p}} \equiv L_{\mathrm{p}} / T_{\mathrm{m}}$, as a function of field. We find that $\Delta S_{\mathrm{p}}$ monotonically decreases with increasing field, as the system approaches the $2 \mathrm{D}$ regime. An important issue is the 
crossover to the $2 \mathrm{D}$ melting regime at very large $B$. Two melting scenarios are possible in two dimensions: a usual 1st order melting and continuous dislocation-mediated melting via the intermediate hexatic phase.37.38 In the first case $\Delta S_{\mathrm{p}}$ has to approach a finite value at $B \rightarrow \infty$ and in the second case it should vanish. Early simulations for a relatively small number $(\lesssim 500)$ of logarithmically interacting particles suggested a 1st order phase transition.24.25.26 However, it is known that to resolve a continuous melting transition in two dimensions, very large systems are required (see, e.g., Ref. 39). Therefore, the nature of the melting transition of $2 \mathrm{D}$ particles with logarithmic interactions is an open issue. Resolving this issue is beyond the scope of this paper.

At low fields the entropy weakly diverges for $B \rightarrow 0$. We understand this as follows: the possible configurations scale as $\sim A / \xi^{2}$, where $\xi^{2}$ is the size of a pancake, and $A$ is the space it can occupy. For the solid state close to the transition the reduced configuration space is $\sim\left\langle u^{2}\right\rangle / \xi^{2}$, because the pancake is confined to an area $A \sim\left\langle u^{2}\right\rangle$. In the liquid the reduced configuration space grows to $\sim a_{0}^{2} / \xi^{2}$, where $a_{0}$ is the average spacing between pancakes. We get thus an entropy difference $\Delta S_{\mathrm{p}} \sim \ln \left(a_{0}^{2} /\left\langle u^{2}\right\rangle\right)$. Since $\left\langle u^{2}\right\rangle$ approaches at low fields a finite field-independent value of the order of $\lambda^{2}$, this explains the observed divergence of $\Delta S_{\mathrm{p}}$ at $B \rightarrow 0$.

For a precise comparison with the experimentally extracted latent heat of vortex-lattice melting 6 one should be careful to include the temperature dependence of $\lambda$, which was shown in Ref. 40 to give extra terms in the observable entropy jump.

\section{CONCLUSIONS}

In this work we have applied a numerical simulation to the suggestion of Ref. 23 to treat the 3D layered pancake system with a mean-field approach for the interlayer interactions, turning the problem into a $2 \mathrm{D}$ system in the presence of a self-consistent substrate potential. In Ref. 23 this substrate model was studied with semianalytic methods. The 2-vertex self-consistent harmonic approximation (2VSCHA) was used to estimate the instability line of the pancake lattice. Also the melting line was estimated by comparing the elastic free energy of the lattice within the substrate model to the free energy of independent 2D liquids, taken from numerical simulations.

Here we have presented results from full numerical simulations of the substrate model. We have directly calculated both the instability and melting lines. Our result for the instability line has a very similar field-dependence to that of the 2VSCHA, which we find to slightly overestimate the instability temperature. The melting line from the semi-analytic approach agrees within error bars to our simulation results for the melting of an intermediate configuration. This implies that the approximations used in Ref. 23 for the free energies of solid and liquid must be extremely good. We have computed the pan- cake fluctuation width $\left\langle u^{2}\right\rangle$ and showed how it varies as a function of temperature: the variation is significantly non-linear below the melting transition, as predicted in Ref. 23. We also calculate the entropy jump across the melting transition, which diverges weakly towards small fields and large melting temperatures.

While we have found a satisfying agreement between our results here and the earlier approximate work of Ref. 23, the true motivation of this project are the possible extensions that can be studied. There is now the exciting prospect to study this pancake vortex system in the presence of pinning disorder.This has been a controversial topic in recent years $41,42,43,44$ that our method should bring some clarity to.

Our results cannot be directly compared with experiments in available layered superconductors because even in the most anisotropic BSCCO the Josephson coupling is not negligible. However, the position of the melting line without the Josephson coupling provides a convenient reference allowing one to understand the role of the Josephson coupling in stabilizing the crystalline phase. In particular, it seems that at low fields even a very small Josephson coupling such as in BSCCO gives a large upward shift to the melting temperature. We also note that it is possible to suppress the effective Josephson coupling by applying a strong in-plane field, as was done in Ref.45, bringing the melting line closer to the "Josephson-free" location. By extending the model to use several layers, it is possible to include Josephson coupling between them, which would realistically describe an anisotropic layered high-temperature superconductor.

\section{ACKNOWLEDGEMENTS}

HF thanks Jacek Generowicz for helpful discussions and EPSRC for financial support. In Argonne this work was supported by the U.S. DOE, Office of Science, under contract \# W-31-109-ENG-38. MJWD is supported by an EPSRC Advanced Fellowship AF/99/0725.

\section{APPENDIX A: CORRELATION CORRECTION TO FREE ENERGY}

The correlation correction to the pancake energy, neglected within the mean-field approach, is given by

$$
\delta \mathcal{E}=\frac{1}{2} \sum_{n \neq n^{\prime}} \int \mathrm{d}^{2} r \mathrm{~d}^{2} r^{\prime} U\left(\mathbf{r}-\mathbf{r}^{\prime}, n-n^{\prime}\right) \delta \rho_{n}(\mathbf{r}) \delta \rho_{n^{\prime}}\left(\mathbf{r}^{\prime}\right)
$$

The correction to the free energy due to this term is given up to second order by

$$
\delta \mathcal{F} \approx\langle\delta \mathcal{E}\rangle_{0}-\frac{\left\langle\delta \mathcal{E}^{2}\right\rangle_{0}-\langle\delta \mathcal{E}\rangle_{0}^{2}}{2 T}
$$

where $\langle\ldots\rangle_{0}$ implies the mean-field averaging. Substituting Eq. (A1) in the last equation and noting that 
$\langle\delta \mathcal{E}\rangle_{0}=0$ we derive for the free energy correction per pancake, $\delta f \equiv a^{2} \delta \mathcal{F} /\left(L_{x} L_{y} N\right)$,

$$
\begin{aligned}
& \delta f \approx-\frac{a^{2}}{4 T L_{x} L_{y}} \sum_{n \neq 0} \int \mathrm{d}^{2} r \mathrm{~d}^{2} r^{\prime} \int \mathrm{d}^{2} r_{1} \mathrm{~d}^{2} r_{1}^{\prime} U\left(\mathbf{r}-\mathbf{r}^{\prime}, n\right) \\
& \times U\left(\mathbf{r}_{1}-\mathbf{r}_{1}^{\prime}, n\right)\left\langle\delta \rho(\mathbf{r}) \delta \rho\left(\mathbf{r}_{1}\right)\right\rangle_{0}\left\langle\delta \rho\left(\mathbf{r}^{\prime}\right) \delta \rho\left(\mathbf{r}_{1}^{\prime}\right)\right\rangle_{0}
\end{aligned}
$$

where $N$ is the total number of layers, $L_{x} L_{y}$ is the layer area, and $\left\langle\delta \rho(\mathbf{r}) \delta \rho\left(\mathbf{r}_{1}\right)\right\rangle_{0} \equiv\left\langle\delta \rho_{n}(\mathbf{r}) \delta \rho_{n}\left(\mathbf{r}_{1}\right)\right\rangle_{0}$ is the den- sity correlation function inside one layer. In the next step we introduce notation for the sum

$$
W\left(\mathbf{r}, \mathbf{r}_{1}\right) \equiv \sum_{n \neq 0} U(\mathbf{r}, n) U\left(\mathbf{r}_{1}, n\right)
$$

for which, using the mixed representation for the interlayer magnetic interaction, $U\left(k_{\perp}, n\right)=$ $-\frac{2 \pi s^{2} \varepsilon_{0}}{\lambda^{2} k_{\perp}^{2}} \frac{\exp \left(-n s \sqrt{\lambda^{-2}+k_{\perp}^{2}}\right)}{\sqrt{\lambda^{-2}+k_{\perp}^{2}}}$, we obtain the formula

$$
W\left(\mathbf{r}, \mathbf{r}_{1}\right)=\frac{s^{3} \varepsilon_{0}^{2}}{2 \pi^{2} \lambda} \int \mathrm{d}^{2} k \mathrm{~d}^{2} k_{1} \frac{\exp \left(i \mathbf{k} \cdot \mathbf{r}+i \mathbf{k}_{1} \cdot \mathbf{r}_{1}\right)}{k^{2} k_{1}^{2} \sqrt{1+\lambda^{2} k^{2}} \sqrt{1+\lambda^{2} k_{1}^{2}}\left(\sqrt{1+\lambda^{2} k^{2}}+\sqrt{1+\lambda^{2} k_{1}^{2}}\right)}
$$

allowing us to represent $W\left(\mathbf{r}, \mathbf{r}_{1}\right)$ in a scaling form

$$
W\left(\mathbf{r}, \mathbf{r}_{1}\right)=\frac{s^{3} \varepsilon_{0}^{2}}{\lambda} w\left(\mathbf{r} / \lambda, \mathbf{r}_{1} / \lambda\right)
$$

By also using a scaling representation for the in-plane density correlation function, $\left\langle\delta \rho(\mathbf{r}) \delta \rho\left(\mathbf{r}_{1}\right)\right\rangle_{0}=\frac{1}{a^{4}} h\left(\mathbf{r} / a, \mathbf{r}_{1} / a\right)$, we derive the scaling representation for the free energy correction

$$
\delta f=-s \varepsilon_{0} \frac{s \varepsilon_{0}}{4 T} \frac{s}{\lambda} \mathcal{G}\left[\frac{a}{\lambda}, \frac{2 s \varepsilon_{0}}{T}\right]
$$

where

$$
\mathcal{G}\left[\frac{a}{\lambda}, \frac{2 s \varepsilon_{0}}{T}\right] \equiv \frac{1}{L_{x} L_{y} a^{6}} \int \mathrm{d}^{2} r \mathrm{~d}^{2} r^{\prime} \mathrm{d}^{2} r_{1} \mathrm{~d}^{2} r_{1}^{\prime} w\left(\frac{\mathbf{r}-\mathbf{r}^{\prime}}{\lambda}, \frac{\mathbf{r}_{1}-\mathbf{r}_{1}^{\prime}}{\lambda}\right) h\left(\mathbf{r} / a, \mathbf{r}_{1} / a\right) h\left(\mathbf{r}^{\prime} / a, \mathbf{r}_{1}^{\prime} / a\right)
$$

is a dimensionless function of the order unity. The meanfield free energy per pancake, $f_{\mathrm{MF}}$, has the scaling property,

$$
f_{\mathrm{MF}}=s \varepsilon_{0} G\left[\frac{a}{\lambda}, \frac{2 s \varepsilon_{0}}{T}\right]
$$

Therefore the free energy correction due to interlayer correlations A2 is smaller than the main term by the factor $s / \lambda$. In particular, the correlation correction shifts the melting temperature up as

$$
\delta T_{\mathrm{m}}=\frac{\delta f_{\mathrm{liq}}-\delta f_{\mathrm{cr}}}{\Delta S}
$$

where $\delta f_{\text {liq }}\left(\delta f_{\text {cr }}\right)$ is the correlation correction to the liquid (crystal) free energy at the melting point and $\Delta S$ is the melting entropy jump. In principle, the mean-field simulations allow to compute the correlation correction and the corresponding shift of the melting temperature. However this computation includes the numerical evaluation of a quite cumbersome integral in Eq. A3.
1 S. N. Artemenko and A. N. Kruglov, Phys. Lett. A 143, 485 (1990).

2 M. V. Feigel'man, V. B. Geshkenbein, and A. I. Larkin, Physica C 167, 177 (1990).

3 A. Buzdin and D. Feinberg, J. Phys. (France) 51, 1971 (1990).

4 J. R. Clem, Phys. Rev. B 43, 7837 (1991).

${ }^{5}$ G. Blatter, M. V. Feigel'man, V. B. Geshkenbein, A. I.
Larkin, and V. Vinokur, Rev. Mod. Phys. 66, 1125 (1994).

6 E. Zeldov, D. Majer, M. Konczykowski, V. B. Geshkenbein, V. M. Vinokur, and H. Shtrikman, Nature 375, 373 (1995).

7 A. Schilling, R. A. Fisher, N. E. Phillips, U. Welp, D. Dasgupta, W. K. Kwok, and G. W. Crabtree, Nature 382, 791 (1996).

8 S. Ryu and D. Stroud, Phys. Rev. B 54, 1320 (1996), cond- 
mat/9602076

9 H. Nordborg and G. Blatter, Phys. Rev. Lett. 79, 1925 (1997), cond-mat/9612025.

10 N. K. Wilkin and H. J. Jensen, Phys. Rev. Lett. 79, 4254 (1997), cond-mat/9705271.

11 H. Nordborg and G. Blatter, Phys. Rev. B 58, 14556 (1998), cond-mat/9803041.

12 A. van Otterlo, R. T. Scalettar, and G. T. Zimányi, Phys. Rev. Lett. 81, 1497 (1998), cond-mat/9803021.

13 C. J. Olson, R. T. Scalettar, G. T. Zimányi, and N. Grønbech-Jensen, Phys. Rev. B 62, R3612 (2000), condmat/9909454.

14 S. Sengupta, C. Dasgupta, H. R. Krishnamurthy, G. I. Menon, and T. V. Ramakrishnan, Phys. Rev. Lett. 67, 3444 (1991).

15 G. I. Menon, C. Dasgupta, H. R. Krishnamurthy, T. V. Ramakrishnan, and S. Sengupta, Phys. Rev. B 54, 16192 (1996).

16 P. S. Cornaglia and C. A. Balseiro, Phys. Rev. B 61, 784 (2000), cond-mat/9904183.

17 D. Reefman and H. B. Brom, Physica 183C, 212 (1991).

18 A. B. Kolton, D. Domínguez, and N. Grønbech-Jensen, Physica C 341-348, 1007 (2000), cond-mat/0002017.

19 A. B. Kolton, D. Domínguez, C. J. Olson, and N. Grønbech-Jensen, Phys. Rev. B 62, R14657 (2000), cond-mat/0004059.

20 C. J. Olson and N. Grønbech-Jensen, Physica C 341-348, 1083 (2000), cond-mat/0002064.

21 C. J. Olson, G. T. Zimányi, A. B. Kolton, and N. Grønbech-Jensen, Phys. Rev. Lett. 85, 5416 (2000), cond-mat/0004054.

22 C. J. Olson, C. Reichhardt, and V. M. Vinokur (2001), cond-mat/0010002.

23 M. J. W. Dodgson, A. E. Koshelev, V. B. Geshkenbein, and G. Blatter, Phys. Rev. Lett. 84, 2698 (2000), condmat/9911439.

24 S. W. de Leeuw and J. W. Perram, Physica 113A, 546 (1982).

25 J. M. Caillol, D. Levesque, J. J. Weis, and J. P. Hansen, Journal of Statistical Physics 28, 325 (1982).

26 P. Choquard and J. Clerouin, Phys. Rev. Lett. (1983).

27 We define the Fourier transform for a function $f(\mathbf{r})$ de- pending on the two-dimensional vector $\mathbf{r}$ as $f(\mathbf{q})=$ $\int \mathrm{d}^{2} r f(\mathbf{r}) \exp (-i \mathbf{q} \cdot \mathbf{r})$ and its inverse transformation as $f(\mathbf{r})=(2 \pi)^{-2} \int \mathrm{d}^{2} q f(\mathbf{q}) \exp (i \mathbf{q} \cdot \mathbf{r})$.

28 M. Frigo and S. G. Johnson, in 1998 ICASSP conference proceedings (1998), vol. 3, pp. 1381-1384.

29 A. E. Koshelev and V. M. Vinokur, Phys. Rev. Lett. 73, 3580 (1994).

30 H. Fangohr, S. J. Cox, and P. A. J. de Groot, Phys. Rev. B 64, 064505 (2001), cond-mat/0104455.

31 H. Fangohr, A. Price, S. Cox, P. A. J. de Groot, G. J. Daniell, and K. S. Thomas, J. Comput. Phys. 162, 372 (2000), physics/0004013.

32 See http://www.soton.ac.uk/ fangohr/preprint/substrate for animations of the freezing and melting process.

33 N. Grønbech-Jensen, Intern. J. Mod. Phys. C 7, 873 (1996).

34 G. Blatter, V. Geshkenbein, A. Larkin, and H. Nordborg, Phys. Rev. B 54, 72 (1996), supr-con/9507003.

35 M. J. W. Dodgson, V. B. Geshkenbein, M. Feigel'man, and G. Blatter (2000), cond-mat/0007072.

36 L. N. Bulaevskii, S. V. Meshkov, and D. Feinberg, Phys. Rev. B 43, 3728 (1991).

37 D. R. Nelson and B. I. Halperin, Phys. Rev. B 19, 2457 (1979).

38 A. P. Young, Phys. Rev. B 19, 1855 (1979).

39 C. Udink and J. van der Elsken, Phys. Rev. B 35, 279 (1987).

40 M. J. W. Dodgson, V. B. Geshkenbein, H. Nordborg, and G. Blatter, Phys. Rev. Lett. 80, 837 (1998), condmat/9705220.

41 B. Khaykovich, E. Zeldov, D. Majer, T. W. Li, P. H. Kes, and M. Konczykowski, Phys. Rev. Lett. 76, 2555 (1996).

42 T. Giamarchi and P. Le Doussal, Phys. Rev. B 55, 6577 (1997), cond-mat/9609112.

43 B. Horovitz and T. R. Goldin, Phys. Rev. Lett. 80, 1734 (1998), cond-mat/9711194.

44 A. E. Koshelev and V. M. Vinokur, Phys. Rev. B 57, 8026 (1998), cond-mat/9801144.

45 J. Mirkovic, S. E. Savel'ev, E. Sugahara, and K. Kadowaki, Phys. Rev. Lett. 86, 886 (2001), cond-mat/0006095. 